\title{
Combination of contrast-enhanced ultrasound and strain elastography to assess cytologically non-diagnostic thyroid nodules
}

\author{
ZI-MEI LIN, QING WEN, CAO-XIN YAN, MIN-QIANG PAN, \\ GUO-QIANG MO, JI-FAN CHEN and PIN-TONG HUANG
}

\begin{abstract}
Department of Ultrasound, The Second Affiliated Hospital, Zhejiang University School of Medicine, Hangzhou, Zhejiang 310009, P.R. China
\end{abstract}

Received April 9, 2019; Accepted September 6, 2019

DOI: $10.3892 / \mathrm{ol} .2019 .11058$

\begin{abstract}
The aim of the present study was to investigate the benefits of combining contrast-enhanced ultrasound (CEUS) and strain elastography (SE) for the diagnosis of thyroid nodules with non-diagnostic fine-needle aspiration cytology (FNAC) results. Between October 2013 and March 2017, CEUS and SE were performed in 226 patients (236 thyroid nodules) with non-diagnostic FNAC results prior to thyroidectomy. The diagnostic value of CEUS, SE and their combination (CEUS+SE) in distinguishing malignant from benign thyroid nodules was evaluated, using surgical pathology as a reference. Receiver operating characteristic curve analysis was used to assess the diagnostic performance of CEUS, SE and CEUS+SE in determining malignant thyroid nodules. Subsequently, the sensitivity, specificity, positive predictive value (PPV), negative predictive value (NPV) and accuracy of CEUS, SE and CEUS + SE were calculated. The malignancy rate in patients with thyroid nodules and non-diagnostic FNAC results was $26.3 \%$ in the present study. The sensitivity, specificity, PPV, NPV, accuracy and area under the curve in predicting malignant thyroid nodules were $80.6,85.6,66.7,92.5,84.3$ and $0.831 \%$, respectively, using SE alone; 59.7, 95.9, 84.1, 86.9, 86.4 and $0.778 \%$, respectively, using CEUS alone; and 83.9, 89.1, 73.6, 94.5, 88.1 and 0.865\%, respectively, using the combination of CEUS and SE. Overall, the combination of CEUS with SE resulted in higher sensitivity, NPV and accuracy in the diagnosis of cytologically non-diagnostic thyroid nodules compared with CEUS or SE alone.
\end{abstract}

\section{Introduction}

The most common type of thyroid cancer is papillary thyroid cancer (PTC), which has a good overall prognosis with a

Correspondence to: Dr Pin-Tong Huang, Department of Ultrasound, The Second Affiliated Hospital, Zhejiang University School of Medicine, 88 Jiefang Road, Hangzhou, Zhejiang 310009, P.R. China E-mail: huangpintong@zju.edu.cn

Key words: contrast-enhanced ultrasound, fine-needle aspiration, thyroid nodule, ultrasound, strain elastography
10 -year survival rate $>90 \%$ (1). Studies have increasingly focused on the overdiagnosis and overtreatment of thyroid disease worldwide $(2,3)$. A study published in New Zealand in 2016 showed that thyroid cancer is overdiagnosed worldwide, suggesting that these tumors do not result in symptoms or death (4). However, a small percentage of patients experience a more aggressive disease, including extrathyroidal extension, clinical lymph node metastasis and distant metastasis. The Thyroid Imaging Reporting and Data System (TIRADS) is helpful in differentiating thyroid nodules by offering a risk stratification model (5). Depending on a constellation or number of suspicious ultrasound (US) features, fine-needle aspiration cytology (FNAC) is recommended (6,7). However, the non-diagnostic rate of thyroid FNAC ranges from $5-20 \%$ (8). The risk of malignancy following a non-diagnostic FNAC result is estimated to be from 1.7-6.6\% (9). Repeated FNAC is recommended for clinical management in cases of non-diagnostic aspirates and can produce satisfactory results in most cases $(10,11)$. However, non-diagnostic results are obtained again in as many as $50 \%$ of second-repeated FNAC analyses (12). A thyroid nodule with non-diagnostic FNAC results raises the controversial question of whether a diagnostic thyroidectomy should be performed.

According to TIRADS, various sonographic features of a thyroid nodule, including the presence of a solid nodule, hypoechogenicity, irregular margins, microcalcifications, a taller-than-wide shape and cervical lymph node metastasis, were associated with an increased likelihood of malignancy. However, no single sonographic feature or combination of features is adequately sensitive or specific for identifying all malignant nodules. Contrast-enhanced ultrasound (CEUS) and strain elastography (SE) are adjunct US imaging techniques that are used to differentiate benign from malignant thyroid nodules in addition to TIRADS. Recently, some prospective studies have confirmed the high predictive value of CEUS and elastography in identifying malignancy $(13,14)$. Furthermore, a study demonstrated that CEUS combined with real-time elastography (RTE) could significantly increase the diagnostic performance for the differential diagnosis of malignant and benign thyroid nodules compared with that of CEUS or RTE alone (15). However, no reports have evaluated thyroid nodules with non-diagnostic FNAC results using a combination of these two technologies. Therefore, the present 
study investigated the effects of combining CEUS and SE on the diagnosis of thyroid nodules with non-diagnostic FNAC results by comparing imaging findings with postoperative histological results.

\section{Materials and methods}

Patients. The present study was conducted between October 2013 and March 2017. Based on the number of suspicious US features that were associated with the nodule (solid composition without cystic components, hypoechogenicity/marked hypoechogenicity, microcalcifications, lobulated or ill-defined margins, and a taller-than-wide shape), each nodule was classified according to the TIRADS as: TIRADS 3 , no suspicious features; TIRADS 4A, one suspicious feature; TIRADS 4B, two suspicious features; TIRADS 4C, three or four suspicious features; or TIRADS 5, five suspicious features (16). Thyroid nodule diagnostic FNAC was recommended for nodules with one or more suspicious sonographic features. All nodules subjected to FNAC were assessed. The ethics committee of The Second Affiliated Hospital Zhejiang University School of Medicine approved this study, and all subjects provided written informed consent prior to their examinations.

A total of 247 patients with 260 nodules and non-diagnostic FNAC results were recruited in the present study. Patients $(\mathrm{n}=21 ; 24$ nodules) who did not undergo thyroidectomy were excluded from the data analysis. Finally, 236 nodules from 226 patients were analyzed. The sizes of the thyroid nodules ranged from 10-28 $\mathrm{mm}$. The patients' ages ranged between 18 and 71 years (mean \pm standard deviation, $55.9 \pm 14.7$ years), and $38.5 \%(\mathrm{n}=87)$ of the patients were male.

Equipment and contrast agent. Conventional US and SE examinations were performed using a 5-13 MHz transducer (Esaote MyLab 90; The Esaote Group). For CEUS, another 3-9 MHz transducer (Esaote MyLab 90; Esaote Group) equipped with contrast-specific, continuous-mode software was used. Sulfur hexafluoride (SonoVue ${ }^{\circledR}$; Bracco Imaging SpA) was used as the US contrast agent.

Performance of conventional US, SE and CEUS. Conventional US, SE and CEUS of the thyroid nodules were performed by two radiologists (Dr. PH and Dr. QW both with $>15,10$ and 4 years of experience in US, SE, and CEUS, respectively). Each patient lay in the supine position with the neck exposed for the US examination. The nodules were all classified according to the TIRADS, based on the number of suspicious features present in each nodule.

SE was performed with light pressure while maintaining the probe perpendicular to the skin surface and stationery for several seconds to allow the acquisition of good quality elastic images with green marks on the screen. Images were displayed in a split-screen mode, with the conventional US images of the nodule with the region of interest including the nodule and sufficient surrounding thyroid tissue (B-mode images) on the right and the elastography images superimposed on the corresponding gray-scale US image on the left, and the tissue stiffness was displayed by a continuum of colors from green (soft tissue) to red (hard tissue).
CEUS was performed following the SE examination on the same or following day. An L522 (3-9 MHz) linear array transducer with an acoustic pressure of $60 \mathrm{kPa}$ was used in each patient. The mechanical index (MI; 0.05-0.07) was automatically selected by the system according to the beam-focus depth. The contrast agent SonoVue ${ }^{\circledR}$ was reconstituted by adding $0.9 \%$ saline $(5 \mathrm{ml})$ and gently shaking the vial by hand to form a homogeneous microbubble suspension. A 19-gauge catheter was inserted into the antecubital fossa vein, and SonoVue ${ }^{\circledR}$ was administered as a bolus injection $(1.2 \mathrm{ml})$, followed immediately by a $10 \mathrm{ml}$ of saline flush via a three-way port in each contrast study. The entire movie sequence (at least $3 \mathrm{~min}$ ) was stored on magnetic optical disks for analysis.

Image interpretation. The SE images were evaluated by two radiologists and classified into five different patterns according to the 5-point Rago scoring system (17) as follows: a score of 1 indicated even elasticity throughout the whole nodule; a score of 2 indicated elasticity in a large part of the nodule; a score of 3 indicated elasticity only at the periphery of the nodule; a score of 4 indicated no elasticity in the nodule; and a score of 5 indicated no elasticity in the nodule or the area with posterior shadowing.

The two radiologists also analyzed the video clips from CEUS. The CEUS features at peak enhancement were summarized as follows: i) A shape enhancement was classified as regular or irregular based on the shapes observed following the contrast agent injection; ii) a margin enhancement was defined as clear or unclear based on the clarity of the margins between the lesion and peripheral tissue; iii) an area enhancement was defined as $<50$ or $\geq 50 \%$ based on the area of the enhanced part/lesion section at the peak enhancement; iv) the type of enhancement included homogeneous enhancement (relative homogeneous diffuse enhancement of the lesions) and heterogeneous enhancement (diffuse enhancement with non-homogeneous or regional microvesicle distribution); and v) the degree of enhancement was characterized as low, equal or high intensity compared with the surrounding thyroid parenchyma $(18,19)$. The definitions of the SE and CEUS patterns are summarized in Table I.

On CEUS, based on the results of previous studies $(20,21)$, nodules with more than one suspicious feature (including low enhancement; heterogeneous and homogeneous) were considered as malignant thyroid nodules. On SE, elasticity scores from 3-5 were considered as the diagnostic criterion for malignant nodules. The patients were diagnosed using the following methods with the combined CEUS and SE: CEUS- and SE-positive or either CEUS- or SE-positive results indicated malignancy, whereas CEUS- and SE-negative results indicated that the lesion was benign.

Pathological examination. All cytological diagnoses were recorded using the Bethesda System for Reporting Thyroid Cytopathology (22) by the pathologist at the Department of Pathology (The Second Affiliated Hospital, Zhejiang University School of Medicine, Hangzhou, China) with $>10$ years of experience in thyroid cytology. The specimens were smeared onto glass slides (3-4 $\mu \mathrm{m}$ ), fixed with 95\% ethyl alcohol (for $4 \mathrm{~h}$ at room temperature), and stained with Diff-Quik and Papanicolaou at room temperature in the following steps: i) The 
Table I. SE scores and CEUS scores.

\begin{tabular}{lll}
\hline Score & \multicolumn{1}{c}{ SE patterns } & \multicolumn{1}{c}{ CEUS patterns } \\
\hline 1 & $\begin{array}{l}\text { Elasticity in the whole nodule: Homogeneously } \\
\text { green }\end{array}$ & $\begin{array}{l}\text { Shape enhancement: Regular or irregular based on the shapes } \\
\text { observed following injection of contrast agent } \\
\text { Margin enhancement: Clear or unclear based on the clarity } \\
\text { of the margin between the lesion and peripheral tissue }\end{array}$ \\
$\begin{array}{l}\text { Elasticity in a large part of the nodule: } \\
\text { Predominantly in green with a few blue areas or } \\
\text { spots }\end{array}$ & $\begin{array}{l}\text { Elasticity only at the peripheral part of the nodule: } \\
\text { Predominantly red with few green areas or spots }\end{array}$ & $\begin{array}{l}\text { Area enhancement: }<50 \text { or } \geq 50 \% \text { based on the area of } \\
\text { the enhancement part/the lesion section at the peak } \\
\text { enhancement } \\
\text { Type of enhancement: Homogeneous (relative homogeneous } \\
\text { diffuse enhancement in lesions), and heterogeneous (diffuse } \\
\text { enhancement presenting non-homogeneous or regional } \\
\text { microvesicle distribution) }\end{array}$ \\
& $\begin{array}{l}\text { No elasticity in the nodule: Completely red } \\
\text { Enhancement degree: Low, equal or high intensity when } \\
\text { compared with the surrounding thyroid parenchyma based on } \\
\text { time-intensity curves }\end{array}$ \\
\hline
\end{tabular}

SE, strain elastography; CEUS, contrast-enhanced ultrasound.

smear was immersed in $75 \%$ alcohol for $1 \mathrm{sec}$; ii) stained with hematoxylin for 3-5 min; iii) immersed in 1.5\% hydrochloric acid for 5-10 sec; iv) washed with water; v) immersed in $75 \%$ then $95 \%$ alcohol for $10 \mathrm{sec}$ each time; iv) immersed in orange green/eosin azure solution for 3-5 min; vii) immersed in $95 \%$ alcohol and anhydrous alcohol; and viii) immersed in xylene solution, wet-sealed and observed with $10 \mathrm{x}$ and $20 \mathrm{x}$ objective light microscope. The remainder of the material was rinsed in saline for processing as a cell block, to aid in DNA testing if required (data not shown). All the 236 nodules were classified as Bethesda III-IV and were included in the present study. The final result was based on histology.

Statistical analysis. The SPSS software package (version 13.0; SPSS Inc.) was used for the statistical analysis of data. The agreement regarding the nodule natures between the two pathologists ( $\mathrm{PH}$ and $\mathrm{QW}$ ) was quantified using the kappa statistic. A kappa statistic $>0.6$ was considered indicative of moderate agreement (23). Disagreements were resolved by consensus or arbitration by a third observer (DJR or DMR). The diagnostic sensitivity, specificity, positive predictive value (PPV) and negative predictive value (NPV) of SE, CEUS, and the combination of SE and CEUS were also assessed by receiver operating characteristic (ROC) curves. $\mathrm{P}<0.05$ was considered to indicate a statistically significant difference.

\section{Results}

The US features. In patients with non-diagnostic FNAC results, the US features of irregular shape, aspect ratio $\geq 1$ (taller-than-wide), and ill-defined margin significantly differed between the malignant and benign nodules. In addition, the features of heterogeneous appearance, near capsular location and a higher elastic score, which are not recommended by TIRADS, also significantly differed between the malignant and benign nodules (Table II). These positive features might
Table II. Ultrasound features.

\begin{tabular}{|c|c|c|c|}
\hline \multirow[b]{2}{*}{ Ultrasound features } & \multicolumn{2}{|c|}{$\begin{array}{c}\text { Pathological results, } \\
\mathrm{n}(\%)\end{array}$} & \multirow[b]{2}{*}{ P-value } \\
\hline & Malignant & Benign & \\
\hline Echogenicity & & & 0.362 \\
\hline Hyperechogenicity & $9(16.1)$ & 47 (83.9) & \\
\hline Hypoechogenicity & $39(31.2)$ & $86(68.8)$ & \\
\hline Isoechogenicity & $14(25.4)$ & $41(74.6)$ & \\
\hline Calcification & & & 0.603 \\
\hline With & $33(27.0)$ & $89(73.0)$ & \\
\hline Without & $29(25.4)$ & 85 (74.6) & \\
\hline Shape & & & 0.001 \\
\hline Regular & $23(16.0)$ & $121(84.0)$ & \\
\hline Irregular & $39(42.4)$ & $53(57.6)$ & \\
\hline Elasticity score & & & $<0.001$ \\
\hline$>3$ & $40(83.3)$ & $8(16.7)$ & \\
\hline$\leq 3$ & $22(11.7)$ & $166(88.3)$ & \\
\hline Aspect ratio & & & $<0.001$ \\
\hline$<1$ & $30(18.1)$ & $136(81.9)$ & \\
\hline$\geq 1$ & $32(45.7)$ & $38(54.3)$ & \\
\hline Margin & & & $<0.001$ \\
\hline Well-defined & $21(14.2)$ & $127(85.8)$ & \\
\hline Ill-defined & $41(46.6)$ & $47(53.4)$ & \\
\hline Size, $10-28 \mathrm{~mm}$ & $62(26.3)$ & $174(73.7)$ & \\
\hline Echotexture & & & $<0.001$ \\
\hline Heterogeneous & $28(49.1)$ & $29(50.9)$ & \\
\hline Homogeneous & $34(18.9)$ & $145(80.9)$ & \\
\hline Location & & & $<0.001$ \\
\hline Near capsular & $21(30.9)$ & $47(69.1)$ & \\
\hline Far from capsular & $41(24.4)$ & $127(75.6)$ & \\
\hline
\end{tabular}




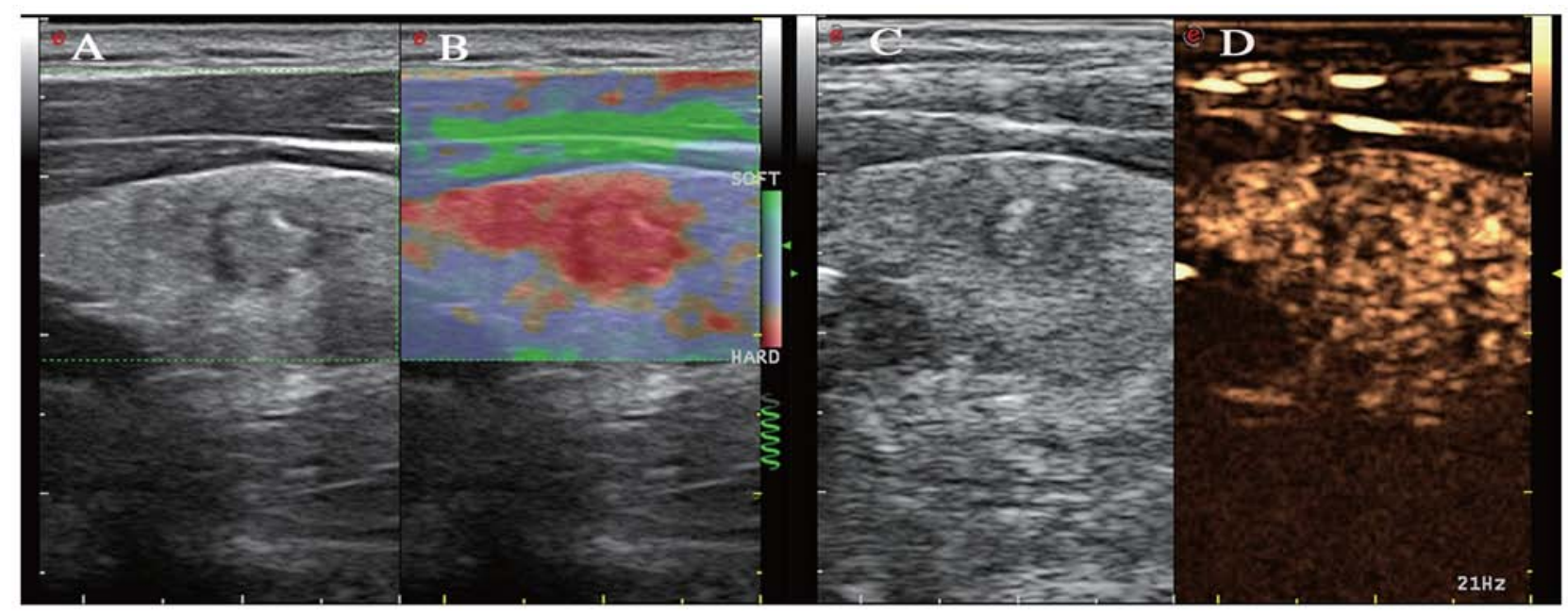

Figure 1. US of a thyroid nodule in a 29-year-old male who underwent fine-needle aspiration. Longitudinal US images demonstrating a hypoechoic nodule in the left thyroid gland. The cytology findings were non-diagnostic, and the pathological diagnosis was papillary carcinoma. (A and B) The SE score was 5, and (C and D) the nodule was hypoenhanced on CEUS. CEUS, contrast-enhanced ultrasound; SE, strain elastography.

explain the high rate of non-diagnostic FNAC results. Based on the TIRADS, $12(5.0 \%), 51(21.6 \%), 137(58.1 \%)$ and 36 $(15.3 \%)$ nodules were classified in categories $4 \mathrm{~A}, 4 \mathrm{~B}, 4 \mathrm{C}$ and 5 , respectively. The rates of malignancy according to TIRADS were $8.3 \%(n=1 / 12), 9.8 \%(n=5 / 51), 27.0 \%(n=37 / 137)$ and $52.8 \%(n=19 / 36)$. The rate of malignancy in patients with initially non-diagnostic FNAC results was $26.3 \%$. The thyroid malignancies with initially non-diagnostic FNAC results included 52 conventional papillary carcinomas, 3 medullary carcinomas and 7 follicular papillary carcinomas. No significant score differences were found between the conventional papillary carcinomas and other carcinomas (data not shown).

The SE and CEUS assessment. The findings from the SE and CEUS showed statistically significant differences in the enhancement margins, shape, enhancement area, intensity and type of enhancement between the benign and malignant thyroid nodules (all $\mathrm{P}<0.01$; data not shown). A total of 53 thyroid carcinoma cases $(85.5 \%)$ had a high elastic score and/or exhibited hypoenhancement. Fig. 1 shows a non-diagnostic nodule with a $5 \mathrm{SE}$ score and hypoenhancement CEUS pattern. The results also indicated that there were significant differences between the malignant and benign nodules with an elasticity score of 1-2 and those with a score of 3-5 ( $\mathrm{P}<0.001$; Table III). Three medullary carcinomas and one follicular carcinoma had a low SE score and were iso- or hyper-enhanced in the present study. The interobserver agreement calculated by the kappa statistic was 0.91 (95\% confidence interval, 0.82-1), indicating high degree of agreement (data not shown).

The ROC analysis. ROC analysis was used to determine whether the findings from the SE and CEUS could help differentiate thyroid carcinoma from benign nodules with non-diagnostic FNAC results. The cut-off value was chosen by the Youden index (specificity + sensitivity -1 ). The ROC curves of CEUS alone, SE alone and the combination of CEUS and SE (SE + CEUS) in the diagnosis of benign and malignant thyroid nodules are shown in Fig. 2. Statistically significant differences in the AUC values were detected among CEUS,
SE and CEUS + SE (all $\mathrm{P}<0.001)$. The comparisons of the sensitivity, specificity, PPV, NPV and accuracy of CEUS alone, SE alone and CEUS + SE for thyroid nodules with non-diagnostic FNAC results are shown in Table III. The sensitivity, specificity, PPV, NPV, accuracy and AUC in predicting malignant thyroid nodules were 80.6, 85.6, 66.7, 92.5, 84.3\% and 0.831 , respectively, for SE alone; 59.7, 95.9, 84.1, 86.9, $86.4 \%$ and 0.778 , respectively, for CEUS alone; and 83.9, 89.1, $73.6,94.5,88.1 \%$ and 0.865 , respectively, for CEUS + SE. The combined SE and CEUS approach had a higher sensitivity, NPV and accuracy than SE or CEUS alone in predicting benign and malignant thyroid nodules with non-diagnostic FNAC results $(\mathrm{P}<0.05)$

\section{Discussion}

Despite the important progress achieved in the field of diagnostic imaging, the preoperative detection of thyroid cancer remains challenging (22). FNAC plays a pivotal role in the diagnosis of thyroid cancer and prevents unnecessary surgery (18). The overall incidence of thyroid cancer is 5.3-28\% in patients with thyroid nodules who undergo FNAC $(24,25)$. Previous studies have shown that the non-diagnostic rate of FNAC for thyroid nodules was between 5 and 19\% $(9,26)$. Although many studies have focused on the overtreatment of thyroid nodules, patients with non-diagnostic FNAC results could benefit from an accurate diagnosis, which could prevent additional stress to the patient caused by the knowledge of the possibility of malignancy.

In the present study, the incidence rate of thyroid tumors in patients with non-diagnostic FNAC results was $26.3 \%$ $(n=62 / 236)$; this rate was higher than that reported in a previous study (27). The possible reasons causing this discrepancy may include but are not limited to several reasons. Firstly, fewer patients were included in these studies compared with the present study. Secondly, some studies did not employ the TIRADS, which is considered the most useful tool for thyroid nodule screening. Most of the patients in the present study (73.4\%) were assigned as categories $4 \mathrm{C}$ and 5 of the TIRADS, 
Table III. Comparison of the sensitivity, specificity, PPV, NPV and accuracy of CEUS alone, SE alone and combination of CEUS $+\mathrm{SE}$ in the non-diagnostic FNAC nodules.

\begin{tabular}{|c|c|c|c|c|c|c|c|c|}
\hline \multirow[b]{2}{*}{ Ultrasound features } & \multicolumn{2}{|c|}{ Pathological results, $\mathrm{n}$} & \multirow[b]{2}{*}{ Sensitivity, \% } & \multirow[b]{2}{*}{ Specificity, \% } & \multirow[b]{2}{*}{ PPV, \% } & \multirow[b]{2}{*}{$\mathrm{NPV}, \%$} & \multirow[b]{2}{*}{ Accuracy, $\%$} & \multirow[b]{2}{*}{ P-value } \\
\hline & Malignant & Benign & & & & & & \\
\hline Elastography & & & 80.6 & 85.6 & 66.7 & 92.5 & 84.3 & $<0.001$ \\
\hline Positive $^{\mathrm{a}}$ & 50 & 25 & & & & & & \\
\hline Negative $^{b}$ & 12 & 149 & & & & & & \\
\hline CEUS & & & 59.7 & 95.9 & 84.1 & 86.9 & 86.4 & $<0.001$ \\
\hline Positive $^{\mathrm{a}}$ & 37 & 7 & & & & & & \\
\hline Negative $^{\mathrm{b}}$ & 25 & 167 & & & & & & \\
\hline $\mathrm{SE}+\mathrm{CEUS}$ & & & 85.5 & 89.1 & 73.6 & 94.5 & 88.1 & $<0.001$ \\
\hline Positive $^{\mathrm{a}}$ & 53 & 19 & & & & & & \\
\hline Negative $^{b}$ & 9 & 155 & & & & & & \\
\hline
\end{tabular}

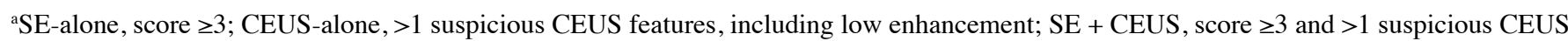
features. ${ }^{\text {bSE }}+$ CEUS, Score $<3$ and non-suspicious CEUS features. PPV, positive predictive value; NPV, negative predictive value; FNAC, fine-needle aspiration cytology; CEUS, contrast-enhanced ultrasound; SE, strain elastography.

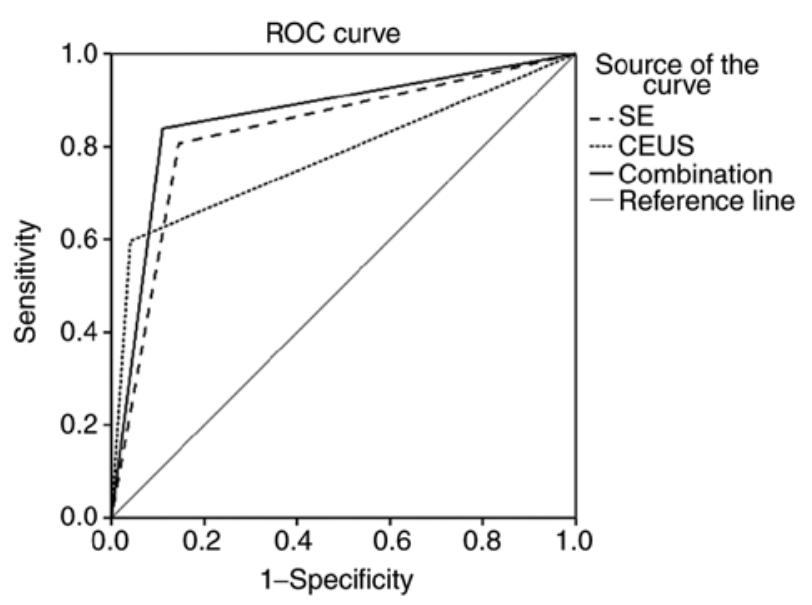

Figure 2. ROC curve of CEUS and SE in determining malignant thyroid nodules. The AUC in predicting malignant thyroid nodules was 0.831 for $\mathrm{SE}$, 0.778 for CEUS, and 0.865 for CEUS + SE, respectively (all $\mathrm{P}<0.001$ ). ROC, receiver operating characteristics; CEUS, contrast-enhanced ultrasound; $\mathrm{SE}$, strain elastography; AUC, area under curve.

which might have caused bias. Thirdly, as shown in the present study, several US features, including calcifications, cystic components, a higher SE score, and near capsular location, may be associated with the increased rate of non-diagnostic FNAC results (Table III); these factors will be considered in future studies. A recent study showed that the prevalence of malignancy in thyroid nodules with indeterminate cytology was $27 \%$ (28). The differences in proportions of these sonographic patterns are thought to explain the observed interinstitutional variability in the risk of malignancy of the indeterminate categories of thyroid cytology. Additionally, only one pathologist reviewed these FNAC slides, and FNAC was performed by multiple radiologists over a long period (between 2013 and 2017). Despite the fact that the present study included patients with pathological confirmation of disease, further attention should be paid to nodules with non-diagnostic FNAC results in future studies.

Several studies suggested that SE and CEUS could differentiate malignant from benign nodules both qualitatively and quantitatively (29-31). However, neither of these two imaging technologies is recommended by the TIRADS, due to concerns regarding operator dependency and reproducibility. Considering the designs of previous studies and the value of SE and CEUS in the present study, the performance of the combination of these two technologies were evaluated in thyroid nodules with non-diagnostic FNAC results.

The use of SE and CEUS, alone or in combination, were investigated in the present study. SE and CEUS are simple and fast to perform, do not require offline strain image reconstruction and may be more practical than other technologies for clinical use. The SE and CEUS findings revealed statistically significant differences in enhancement and elastic features between the benign and malignant nodules. The reason for these different findings may be that malignant tissues are usually harder than benign tissues and that anomalous vascular distribution may be present in malignant nodules. However, not all tumor tissues had increased elastic scores. In total, 9 malignant nodules showed false negative results, and 19 benign nodules were misdiagnosed as malignant tumors. The gross anatomy and cellular patterns of follicular carcinoma overlap with those of benign follicular adenoma, which explains why this type of thyroid malignancy can only be differentiated from benign follicular adenoma when capsular or vascular invasion is observed histologically (32). These findings are consistent with the results reported by Rubaltelli et al (33) and Cantisani et al (34). Another 5 PTCs showed CEUS negative results, which is likely due to the size of the nodule. These PTCs were $>2 \mathrm{~cm}$, and a previous study revealed an association between the nodule size and CEUS enhancement pattern (35).

Recently, Sui et al (15) found that CEUS combined with elastography could significantly increase the diagnostic performance in the differential diagnosis of malignant and 
benign thyroid nodules compared with CEUS or elastography alone. Rago et al (17) evaluated 195 consecutive thyroid nodules in 176 patients with indeterminate or non-diagnostic FNAC results with SE and found a sensitivity of $96.8 \%$ and a specificity of $91.8 \%$ in distinguishing benign and malignant nodules. Similarly, the findings of the present study indicated that the diagnostic power (including the sensitivity, NPV and accuracy) of SE + CEUS proved to be higher than that of either modality alone. The combination of SE and CEUS provides noninvasive imaging of tissue characteristics and an indirect characterization of intranodular vascularization. Most patients of the present study declined repeated FNAC due to anxiety, as the patients wanted to avoid the additional stress caused by the possibility of malignancy. The combination of SE and CEUS might be helpful to patients with non-diagnostic results. If the combination of CEUS and SE shows negative results, a nodule with a suspicious feature could be followed up in a short time, rather than subjecting the patient to biopsy or resection. Therefore, the addition of the combination with a higher negative predictive value could be appropriate for benign non-diagnostic nodules.

One of the limitations of the present study include that the SE and CEUS were performed in patients who were known to be candidates for thyroid FNAC, which might potentially impact the scoring of the nature of the thyroid nodules. Future studies that are based on a completely blinded evaluation are necessary to provide conclusive evidence regarding the roles of SE and CEUS in the diagnosis of thyroid nodules. Secondly, papillary carcinoma and follicular carcinoma differ in clinical aspects and the cytological structure. Although no significant difference was found between conventional papillary carcinomas and follicular papillary carcinomas in the present study, the guidelines for the differentiation of malignancy according to the pathological type should be further studied. Thirdly, only one pathologist reviewed the FNAC slides and the intra- and inter-observer reliability in the interpretation of the cytopathology of thyroid FNA was not determined.

In conclusion, the combination of CEUS and SE has high sensitivity, NPV and accuracy in determining malignant thyroid nodules with cytologically non-diagnostic results compared with CEUS or SE alone.

\section{Acknowledgements}

Not applicable.

\section{Funding}

The present study was supported by the National Natural Science Foundation of China (grant nos. 81527803 and 81420108018).

\section{Availability of data and materials}

The datasets used and/or analyzed during the present study are available from the corresponding author on reasonable request.

\section{Authors' contributions}

PTH and ZML provided administrative support and designed the study. QW, CY, MQP, JC and ZML recruited patients and acquired consumables. MQP, CXY, GM and ZML collected and organized the data. ZML, QW and JC analyzed and interpreted the data. All authors were involved in the conception and design of the study, and wrote and provided final approval of the manuscript for publication.

\section{Ethics approval and consent to participate}

The Ethics Committee of The Second Affiliated Hospital Zhejiang University School of Medicine approved the present study, and all subjects provided written informed consent prior to their examinations.

\section{Patient consent for publication}

All subjects provided written informed consent for publication.

\section{Competing interests}

The authors declare that they have no competing interests.

\section{References}

1. Caron NR and Clark OH: Papillary thyroid cancer. Curr Treat Options Oncol 7: 309-319, 2006.

2. Cronan JJ: Thyroid nodules: Is it time to turn off the us machines? Radiology 247: 602-604, 2008.

3. Jung M: Breast, prostate, and thyroid cancer screening tests and overdiagnosis. Curr Probl Cancer 41: 71-79, 2016.

4. Vaccarella S, Franceschi S, Bray F, Wild CP, Plummer M and Dal Maso L:Worldwide thyroid-cancer epidemic? The increasing impact of overdiagnosis. N Engl J Med 375: 614-617, 2016.

5. Tessler FN, Middleton WD, Grant EG, Hoang JK, Berland LL, Teefey SA, Cronan JJ, Beland MD, Desser TS, Frates MC, et al: ACR thyroid imaging, reporting and data system (TI-RADS): White paper of the ACR TI-RADS committee. J Am Coll Radiol 14: 587-595, 2017.

6. Mittendorf EA, Tamarkin SW and McHenry CR: The results of ultrasound-guided fine-needle aspiration biopsy for evaluation of nodular thyroid disease. Surgery 132: 648-653, 2002.

7. Gharib H, Papini E, Paschke R, Duick DS, Valcavi R, Hegedüs L, Vitti P and AACE/AME/ETA Task Force on Thyroid Nodules: American association of clinical endocrinologists, associazione medici endocrinologi, and european thyroid association medical guidelines for clinical practice for the diagnosis and management of thyroid nodules: Executive summary of recommendations. J Endocrinol Invest 33: 51-56, 2010.

8. Alexander EK, Heering JP, Benson CB, Frates MC, Doubilet PM, Cibas ES and Marqusee E: Assessment of nondiagnostic ultrasound-guided fine needle aspirations of thyroid nodules. J Clin Endocrinol Metab 87: 4924-4927, 2002.

9. Al Maqbali T, Tedla M, Weickert MO and Mehanna H: Malignancy risk analysis in patients with inadequate fine needle aspiration cytology (FNAC) of the thyroid. PLoS One 7: e49078, 2012.

10. Ali SZ: Thyroid cytopathology: Bethesda and beyond. Acta Cytol 55: 4-12, 2011.

11. Baloch Z, LiVolsi VA, Jain P, Jain R, Aljada I, Mandel S, Langer JE and Gupta PK: Role of repeat fine-needle aspiration biopsy (FNAB) in the management of thyroid nodules. Diagn Cytopathol 29: 203-206, 2003.

12. Orija IB, Pineyro M, Biscotti C, Reddy SS and Hamrahian AH: Value of repeating a nondiagnostic thyroid fine-needle aspiration biopsy. Endocr Pract 13: 735-742, 2007.

13. Zhang Y,Zhang MB, Luo YK, Li J,Wang ZL and Tang: The value of peripheral enhancement pattern for diagnosing thyroid cancer using contrast-enhanced ultrasound. Int J Endocrinol 2018: 1625958, 2018.

14. Bojunga $J$ and Mondorf A: Thyroid elastography. Laryngo-Rhino-Otologie 98: 150-156, 2019.

15. Sui X, Liu HJ, Jia HL and Fang QM: Contrast-enhanced ultrasound and real-time elastography in the differential diagnosis of malignant and benign thyroid nodules. Exp Ther Med 12: 783-791, 2016. 
16. Kwak JY, Han KH, Yoon JH, Moon HJ, Son EJ, Park SH, Jung HK, Choi JS, Kim BM and Kim EK: Thyroid imaging reporting and data system for US features of nodules: A step in establishing better stratification of cancer risk. Radiology 260: 892-899, 2011.

17. Rago T, Santini F, Scutari M,Pinchera A and Vitti P: Elastography: New developments in ultrasound for predicting malignancy in thyroid nodules. J Clin Endocrinol Metab 92: 2917-2922, 2007.

18. Zhang B, Jiang YX, Liu JB, Yang M, Dai Q, Zhu QL and Gao P Utility of contrast-enhanced ultrasound for evaluation of thyroid nodules. Thyroid 20: 51-57, 2010.

19. Zhou Q, Xu YB, Jiang J, Ma WQ, Wang H, Li M and Lei XY: Differential diagnostic value of contrast-enhanced ultrasound in calcified thyroid nodules. Zhonghua Er Bi Yan Hou Tou Jing Wai Ke Za Zhi (Chinese) 48: 726-729, 2013.

20. Wang Y, Nie F, Liu T, Yang D, Li Q, Li J and Song A: Revised value of contrast-enhanced ultrasound for solid hypo-echoic thyroid nodules graded with the thyroid imaging reporting and data system. Ultrasound Med Biol 44: 930-940, 2018.

21. Wu Q, Li Y and Wang Y: Diagnostic value of 'absent' pattern in contrast-enhanced ultrasound for the differentiation of thyroid nodules. Clin Hemorheol Microcirc 63: 325-334, 2016.

22. Cibas ES and Ali SZ: The 2017 bethesda system for reporting thyroid cytopathology. Thyroid 27: 1341-1346, 2017.

23. Landis JR and Koch GG: An application of hierarchical kappa-type statistics in the assessment of majority agreement among multiple observers. Biometrics 33:363-374, 1977.

24. Kim EK, Park CS, Chung WY, Oh KK, Kim DI, Lee JT and Yoo HS: New sonographic criteria for recommending fine-needle aspiration biopsy of nonpalpable solid nodules of the thyroid AJR Am J Roentgenol 178: 687-691, 2002.

25. Bessey LJ, Lai NB, Coorough NE, Chen H and Sippel RS: The incidence of thyroid cancer by fine needle aspiration varies by age and gender. J Surg Res 184: 761-765, 2013.

26. Williams BA, Bullock MJ, Trites JR, Taylor SM and Hart RD: Rates of thyroid malignancy by FNA diagnostic category. J Otolaryngol Head Neck Surg 42: 61, 2013.
27. Akgul O, Ocak S, Keskek M, Koc M and Tez M: Risk of malignancy in non-diagnostic thyroid fine-needle aspiration biopsy in multinodular goitre patients. Endocr Regul 45: 9-12, 2011.

28. Valderrabano P, McGettigan MJ, Lam CA, Khazai L, Thompson ZJ, Chung CH, Centeno B and McIver B: Thyroid nodules with indeterminate cytology: Utility of the American thyroid association sonographic patterns for cancer risk stratification. Thyroid 28: 1004-1012,2018.

29. Rago T, Scutari M, Santini F, Loiacono V, Piaggi P, Di Coscio G, Basolo F, Berti P, Pinchera A and Vitti P: Real-time elastosonography: Useful tool for refining the presurgical diagnosis in thyroid nodules with indeterminate or nondiagnostic cytology. J Clin Endocrinol Metab 95: 5274-5280, 2010.

30. Schleder S, Janke M, Agha A, Schacherer D, Hornung M, Schlitt HJ, Stroszczynski C, Schreyer AG and Jung EM: Preoperative differentiation of thyroid adenomas and thyroid carcinomas using high resolution contrast-enhanced ultrasound (CEUS). Clin Hemorheol Microcirc 61: 13-22, 2015.

31. Ma JJ, Ding H, Xu BH, Xu C, Song LJ, Huang BJ and Wang WP: Diagnostic performances of various gray-scale, color doppler, and contrast-enhanced ultrasonography findings in predicting malignant thyroid nodules. Thyroid 24: 355-363, 2014.

32. Trimboli P and Crescenzi A: Thyroid core needle biopsy: Taking stock of the situation. Endocrine 48:779-785, 2015.

33. Rubaltelli L, Corradin S, Dorigo A, Stabilito M, Tregnaghi A, Borsato $\mathrm{S}$ and Stramare R: Differential diagnosis of benign and malignant thyroid nodules at elastosonography. Ultraschall Med 30: 175-179, 2009.

34. Cantisani V, D'Andrea V, Mancuso E, Maggini E, Di Segni M, Olive M, Lodise P, Palermo S, De Antoni S, Redler A, et al: Prospective evaluation in 123 patients of strain ratio as provided by quantitative elastosonography and multiparametric ultrasound evaluation (ultrasound score) for the characterisation of thyroid nodules. Radiol Med 118: 1011-1021, 2013.

35. Yuan Z, Quan J, Yunxiao Z, Jian C and Zhu H: Association between real-time contrast-enhanced ultrasound characteristics and thyroid carcinoma size. Mol Clin Oncol 3: 743-746, 2015. 